International Journal of Difference Equations

ISSN 0973-6069, Volume 14, Number 2, pp. 91-113 (2019)

https://dx.doi.org/10.37622/IJDE/14.2.2019.91-113

\title{
Necessary and Sufficient Conditions for Debt Sustainability. True or a Paradox?
}

\author{
Elias Camouzis and Stelios Kotsios \\ National and Kapodistrian University of Athens \\ Department of Economics \\ 1 Sofokleous Str., Athens, 10559, Greece
}

\begin{abstract}
We present necessary and sufficient conditions such that Public Debt, denoted by $B_{n}$, is sustainable. The government budget constraint equation implies that: Issuing of new debt $B_{n+1}$ plus current tax revenues $\tau_{n+1} Y_{n}$, should balance out current government spending on goods and services $G_{n+1}$ plus the cost of servicing current debt $r_{n} B_{n}$, that is, $B_{n+1}=\left(1+r_{n}\right) B_{n}-\tau_{n+1} Y_{n}+G_{n+1}, \quad n=0,1, \ldots$, where, $r_{n}$ and $\tau_{n+1}$ are: nominal interest rate and taxation rate, respectively, while $\left\{Y_{n}\right\}$ is the nominal GDP sequence. Solutions of the budget constraint equation, might as well be negative and this creates a disturbance in the study of the long time behavior of debt $B_{n}$. We present a system of equations, which contains the budget constraint model as a special case. The two state variables are: Public Debt, $B_{n}$, and savings, $F_{n}$. The values of the solutions of the system are always nonnegative, and this allows for explicit computation of necessary and sufficient conditions for debt sustainability. We also provide several examples, by assuming that the values $Y_{n}$ of nominal GDP satisfy the Samuelson-Hicks model
\end{abstract}

$$
Y_{n+1}=b(1+k) Y_{n}-b k Y_{n-1}+G, \quad n=0,1, \ldots,
$$

where $b \in(0,1)$ is the marginal propensity to consume and $k \in(0, \infty)$ is the acceleration coefficient.

AMS Subject Classifications: 39A10, 39A11.

Keywords: Public debt, Savings, Debt sustainability conditions, GDP, Global Asymptotic stability, Periodic solutions, A-periodic solutions.

Received December 17, 2018; Accepted September 15, 2019

Communicated by Martin Bohner 


\section{Introduction}

In this article, we present necessary and sufficient conditions for public debt sustainability and we also examine the future behavior of Public Debt, namely $B_{n}$, which is assumed to be nonnegative for all nonnegative values of the integer $n$. The set of variables associated with the future behavior of Public Debt are: nominal GDP, $Y_{n}$, government spending, $G_{n}$, interest rate, $r_{n}$, and taxation rate, $\tau_{n}$. Primary surplus or deficit at time $n+1$ depends on nominal GDP, taxation rate, and government spending. More precisely,

$$
S_{n+1}=\tau_{n+1} Y_{n}-G_{n+1}, n=0,1, \ldots .
$$

We assume that, for all $n$,

$$
r_{n}, \tau_{n} \in(0,1), G_{n} \in(0, \infty), S_{n} \in R, \text { and } \liminf _{n \rightarrow \infty} r_{n}>0 .
$$

The equation below, is well known as, budget constraint equation:

$$
B_{n+1}=\left(1+r_{n}\right) B_{n}-S_{n+1}, \quad n=0,1, \ldots .
$$

Assuming positive interest rates public debt increases in the future. Primary surpluses reduce public debt in the future while primary deficits enlarge it. In view of (1.1) and the variables, $d_{n}=\frac{B_{n}}{Y_{n}}$, debt to GDP ratio, $s_{n}=\frac{S_{n}}{Y_{n}}$, surplus to GDP ratio and $g_{n}=$ $\frac{1+Y_{n+1}}{1+Y_{n}}$, growth of the economy, we find the debt to GDP ratio dynamic equation

$$
d_{n+1}=\frac{1+r_{n}}{1+g_{n}} \cdot d_{n}-s_{n+1}, \quad n=0,1, \ldots .
$$

The present value of public debt today, is $B_{0}$. At any future stage, $n$, the present value of public debt is, $B_{n}$, and the present value of all future surpluses or deficits by $P V_{n}$. Provided that the infinite sum exists,

$$
P V_{n}=\sum_{j=0}^{\infty} \frac{S_{j+1}}{\prod_{k=n}^{j}\left(1+r_{k}\right)},
$$

we present the following definition.

Definition 1.1. Public Debt at time $t=n$ is sustainable, if and only if, $B_{n} \leq P V_{n}$.

The following identity, whose proof is straightforward, will be useful in the sequel.

$$
B_{n+1}-P V_{n+1}=\left(1+r_{n}\right)\left(B_{n}-P V_{n}\right), \text { for all } n \geq 0,
$$

The following theorem presents necessary and sufficient conditions for debt sustainability. 
Theorem 1.2. Assume that:

(i) $\left\{r_{n}\right\}$ and $\left\{S_{n}\right\}$ are arbitrary future sequences representing interest rate and surplus, such that, for all nonnegative integer values of $n$,

$$
r_{n} \in(0,1) \text { and } S_{n} \in R \text {. }
$$

(ii) The present value of all future surpluses, is a positive real number, that is,

$$
P V_{0} \in(0, \infty)
$$

(iii) All future debt values, current debt value included, are nonnegative and the budget constraint equation (1.1) is satisfied for all nonnegative integer values of $n$.

Then all debt values, $B_{n}$, current and future, are sustainable, if and only if,

$$
B_{0} \leq P V_{0}
$$

Proof. The proof follows from (1.3) and Definition 1.1.

As far as we know, the existing knowledge in the literature is that debt is sustainable if

$$
B_{0}=P V_{0}
$$

The amount of government debt today is equal with the present value of all future surpluses, and so it can be paid off. However, this is only a sufficient condition for debt sustainability because, if a government can afford a debt equal to the present value of all future surpluses can afford even less. Thus, Condition (1.4), is in line with the knowledge of the existing literature, only sharper. In addition, the result of Theorem 1.2 states that Condition (1.4) is necessary and sufficient so that all future debt values are sustainable. Assuming the validity of the result of Theorem 1.2, one may easily conclude that if a government debt is sustainable today it has to be sustainable in the future as well. This type of argument looks like a paradox. In this article, we present several results which might explain why this type of argument might not be a paradox.

Our investigation starts by challenging the hypotheses of Theorem 1.2. In particular, we focus on the third hypothesis of Theorem 1.2. In other words, we pose the question: Is the result of Theorem 1.2 valid if only the third hypothesis of the theorem is violated? The answer to this question is NO! To see this observe that the solution of (1.1), in closed form is

$$
B_{n}=\prod_{i=0}^{n-1}\left(1+r_{i}\right)\left(B_{0}-\sum_{j=0}^{n-1} \frac{S_{j+1}}{\prod_{t=0}^{j}\left(1+r_{t}\right)}\right) .
$$

As it turns out, if $B_{0}=P V_{0}$, then the result of the theorem is still valid. However, if $B_{0}<P V_{0}$, then for some future $n$, the value of $B_{n}$ is negative, and so, that moment 
in the future, the debt sequence $\left\{B_{n}\right\}$ does not satisfy (1.1). Thus, the third condition of Theorem 1.2 is violated, and the corresponding result is no longer valid. This might be a partial explanation of the fact that the only sustainability condition known in the literature is $B_{0}=P V_{0}$. This type of problem can be easily resolved by introducing a new variable, the variable of savings, as part of national savings, namely $F_{n}$, which assumes nonnegative values for all $n$. Savings at stage $n+1$ is $F_{n+1}$ and might become strictly positive if $S_{n+1}>\left(1+r_{n}\right) B_{n}$. In other words, primary surplus at stage $n+1$ can be used to pay off the debt and the remaining amount can be transferred to savings.

In Section 2, we give a detailed presentation of our model.

\section{The Model}

At time $n \geq 0$, assume that

$$
\begin{gathered}
Y_{n}=\text { national income, } B_{n}=\text { national debt, } F_{n}=\text { savings, } \\
T_{n}=\text { taxes collected by government, } G_{n}=\text { government expenditure, }
\end{gathered}
$$

with initial conditions,

$$
B_{0}, Y_{0}, T_{0}, G_{0}>0 \text { and } F_{0} \geq 0 \text {. }
$$

If, $\tau_{n}$, is the tax rate, then $T_{n+1}=\tau_{n+1} Y_{n}$. Let, $S_{n}=T_{n}-G_{n}$, the difference between collected taxes and government expenditure. If, $T_{n} \geq G_{n}$, it is called surplus, otherwise it is called deficit. Also, $r_{n}$, is the interest rate applied to national debt and, $\theta_{n}$, the interest rate applied to savings, such that,

$$
r_{n}, \theta_{n} \in(0,1) \text {, for all } n \geq 0 .
$$

Finally, $\gamma_{n} \in[0,1]$, is the percentage of savings used to service debt and $p_{n} \in[0,1]$ is the proportion of national debt to be paid off.

If for some $n \geq 0$,

$$
\left(1+r_{n}\right) B_{n}-\left(1+\theta_{n}\right) F_{n}-S_{n+1} \geq 0
$$

that is, debt plus interest is greater than or equal to, savings plus interest and surplus, then

$$
F_{n+1}=\left(1+\theta_{n}\right)\left(1-\gamma_{n}\right) F_{n} \text { and } B_{n+1}=\left(1+r_{n}\right) B_{n}-\gamma_{n}\left(1+\theta_{n}\right) F_{n}-S_{n+1}
$$

On the other hand, if for some $n \geq 0$,

$$
\left(1+r_{n}\right) B_{n}-\left(1+\theta_{n}\right) F_{n}-S_{n+1}<0
$$


that is, if debt plus interest is less than savings plus interest, plus surplus, then

$$
B_{n+1}=\left(1+r_{n}-p_{n}\right) B_{n} \text { and } F_{n+1}=\left(1+\theta_{n}\right) F_{n}+S_{n+1}-p_{n} B_{n},
$$

that is, we pay off a certain percentage, $p_{n} \in[0,1]$, of public debt. The remaining amount, $F_{n+1}$, replaces the amount of savings of the previous period, $F_{n}$.

By setting,

$$
R B_{n}=\left(1+r_{n}\right) B_{n}-\left(1+\theta_{n}\right) F_{n}-S_{n+1},
$$

our study model becomes

$$
\left.\begin{array}{l}
B_{n+1}=\left(1+r_{n}\right) B_{n}-\gamma_{n}\left(1+\theta_{n}\right) F_{n}-S_{n+1} \text { and } F_{n+1}=\left(1+\theta_{n}\right)\left(1-\gamma_{n}\right) F_{n}, \text { if } R B_{n} \geq 0 \\
B_{n+1}=\left(1+r_{n}-p_{n}\right) B_{n} \text { and } F_{n+1}=\left(1+\theta_{n}\right) F_{n}+S_{n+1}-p_{n} B_{n}, \text { if } R B_{n}<0
\end{array}\right\},
$$

$n=0,1, \ldots$, with $B_{0}>0$ and $F_{0} \geq 0$.

Although, in general, $r_{n} \neq \theta_{n}$, in this article, we study the special case of (2.5), in which $r_{n}=\theta_{n}$. In this case, (2.5) becomes,

$$
\left.\begin{array}{l}
B_{n+1}=\left(1+r_{n}\right)\left(B_{n}-\gamma_{n} F_{n}\right)-S_{n+1} \text { and } F_{n+1}=\left(1+r_{n}\right)\left(1-\gamma_{n}\right) F_{n}, \text { if } R B_{n} \geq 0 \\
B_{n+1}=\left(1+r_{n}-p_{n}\right) B_{n} \text { and } F_{n+1}=\left(1+r_{n}\right) F_{n}+S_{n+1}-p_{n} B_{n}, \text { if } R B_{n}<0
\end{array}\right\}
$$

$n=0,1, \ldots$, with $B_{0}>0$ and $F_{0} \geq 0$.

The following amazing theorem needs serious attention.

Theorem 2.1. For all future times, $n \geq 0$, current stage included,

$$
B_{n}, F_{n} \geq 0
$$

and

$$
\left(B_{n+1}-F_{n+1}\right)=\left(1+r_{n}\right)\left(B_{n}-F_{n}\right)-S_{n+1} .
$$

Proof. The proof is straightforward and the details are omitted.

Remark 2.2. In view of the result of Theorem 2.1, it is immaterial what percentage, $\gamma_{n}$, of savings is used to service debt. The same is true for the percentage of debt paid off, namely, $p_{n}$. For convenience, we assume that $\gamma_{n}=1$ and that $p_{n}=r_{n}$. That is, we use the entire amount of savings to service debt and if debt is fully covered by savings and surplus we only pay debt's interest.

In view of the remark above, System (2.6) becomes

$$
\left.\begin{array}{l}
B_{n+1}=\left(1+r_{n}\right)\left(B_{n}-F_{n}\right)-S_{n+1} \text { and } F_{n+1}=0, \text { if } R B_{n} \geq 0 \\
B_{n+1}=B_{n} \text { and } F_{n+1}=\left(1+r_{n}\right) F_{n}+S_{n+1}-r_{n} B_{n}, \text { if } R B_{n}<0
\end{array}\right\} .
$$

Furthermore, we assume that the initial amount of national debt is strictly positive, $B_{0}>0$, and that the initial amount of savings is equal to zero, $F_{0}=0$. 


\section{Necessary and Sufficient Condition for Debt Sustain- ability}

We define debt sustainability in view of our model of study, System (2.8).

Definition 3.1. National Debt at time $t=n$ is sustainable, if and only if, $B_{n} \leq F_{n}+$ $P V_{n}$.

Remark 3.2. Note that Definition 3.1 contains Definition 1.1, as a special case, if the amount of savings is always equal to zero. Indeed, if for all $n, F_{n}=0$, then National Debt at time, $t=n$, is sustainable, if and only if, $B_{n} \leq P V_{n}$.

The following identity, whose proof is straightforward, will be useful in the sequel.

$$
B_{n+1}-F_{n+1}-P V_{n+1}=\left(1+r_{n}\right)\left(B_{n}-F_{n}-P V_{n}\right), \text { for all } n \geq 0,
$$

The following theorem presents necessary and sufficient conditions for debt sustainability.

Theorem 3.3. Assume that $\left\{\left(B_{n}, F_{n}\right)\right\}$ is a paired sequence of values of national debt and savings, which is a solution of System (2.8) and that

(i) $\left\{r_{n}\right\}$ and $\left\{S_{n}\right\}$ are arbitrary future sequences representing interest rate and surplus, such that, for all nonnegative integer values of $n$,

$$
r_{n} \in(0,1) \text { and } S_{n} \in R
$$

(ii) The present value of all future surpluses, is a positive real number, that is,

$$
P V_{0} \in(0, \infty)
$$

Then all debt values, $B_{n}$, current and future, are sustainable, if and only if,

$$
B_{0} \leq P V_{0}
$$

Proof. The proof follows from (3.1) and Definition 3.1.

Remark 3.4. The following Debt sustainability condition in terms of Debt to GDP ratio

$$
d_{0} \leq \sum_{j=0}^{\infty} s_{j+1}\left(\prod_{t=0}^{j} \frac{1+g_{t}}{1+r_{t}}\right)
$$

is equivalent to Condition (3.2). 


\section{Sustainability Scenarios and Future Behavior}

In this section, we analyze some special cases of debt sustainability and we examine the associated future behavior of public debt and savings. Before we proceed we present a lemma which will be useful in the sequel.

Lemma 4.1. Assume that

(i) $\left\{r_{n}\right\}$ is an arbitrary future sequence representing interest rate, such that, for all nonnegative integer values of $n$,

$$
r_{n} \in(0,1) \text { and } \liminf _{n \rightarrow \infty} r_{n}>0
$$

Then

$$
\sum_{j=n}^{\infty} \frac{r_{j}}{\prod_{t=n}^{j}\left(1+r_{t}\right)}=1, \text { for all } n \geq 0
$$

Proof. Set

$$
u_{m, n}=\sum_{j=n}^{m} \frac{r_{j}}{\prod_{t=n}^{j}\left(1+r_{t}\right)}, \quad n=0,1, \ldots, \quad m=n, n+1, \ldots,
$$

and observe that

$$
u_{m, n}=1-\frac{1}{\prod_{t=n}^{m}\left(1+r_{t}\right)} .
$$

For each $n$, By taking limits, as $m \rightarrow \infty$, the result follows. The proof is complete.

A natural question that arises is the following: Is there an arbitrary sequence of positive interest rates and surpluses, such that, savings is always equal to zero and public debt values are always nonnegative? If this is true, then (1.1), which is a special case to our model of study (2.8), is always satisfied. The answer to this question is Yes. In fact, the next theorem establishes that, if for each $n$, future surplus is equal to debt interest, then for all future $n$, national debt is equal to its initial value, $B_{0}$, and savings are equal to zero.

Theorem 4.2. Assume that $\left\{\left(B_{n}, F_{n}\right)\right\}$ is a solution of System (2.8) and that

(i) The initial debt value, $B_{0}$, is an arbitrary positive real number and that the initial value of savings, $F_{0}$ is equal to zero. That is,

$$
B_{0} \in(0, \infty) \text { and } F_{0}=0 .
$$

(ii) $\left\{r_{n}\right\}$ is an arbitrary future sequence representing interest rate, such that, for all nonnegative integer values of $n$,

$$
r_{n} \in(0,1) \text { and } \liminf _{n \rightarrow \infty} r_{n}>0
$$


(iii) For all n, there is a positive surplus which pays off public debt interest. That is,

$$
S_{n+1}=r_{n} B_{n}, \text { for all } n \geq 0 \text {. }
$$

Then all debt values, $B_{n}$, current and future, are sustainable and all savings, $F_{n}$, current and future, are equal to zero. More precisely,

$$
B_{n}=B_{0}=P V_{n}=P V_{0}=\sum_{j=0}^{\infty} \frac{S_{j+1}}{\prod_{t=0}^{j}\left(1+r_{t}\right)} .
$$

Proof. By using induction, one can easily see that, for all future values of $n, F_{n}=0$ and $B_{n}=B_{0}$. Thus, $S_{n+1}=r_{n} B_{n}=r_{n} B_{0}$. The result now follows from (4.1). Indeed,

$$
P V_{n}=\sum_{j=n}^{\infty} \frac{S_{j+1}}{\prod_{t=n}^{j}\left(1+r_{t}\right)}=B_{0} \cdot \sum_{j=n}^{\infty} \frac{r_{j}}{\prod_{t=n}^{j}\left(1+r_{t}\right)}=B_{0}, \text { for all } n \geq 0 .
$$

The proof is complete.

Remark 4.3. Theorem 4.2 presents a scenario in which debt remains constant or stable. In other words, by paying off interest only, in each time period, the debt is always sustainable, equal to its initial value, $B_{0}$. The hypothesis of the theorem, $S_{n+1}=r_{n} B_{n}$, is equivalent to $s_{n+1}=d_{n} \cdot \frac{r_{n}-g_{n}}{1+g_{n}}$, which is known as debt stabilizing balance equation. In other words, for all $n, d_{n+1}=d_{n}+\frac{r_{n}-g_{n}}{1+g_{n}} \cdot d_{n}-s_{n+1}=d_{n}$, that is debt to GDP ratio, is stable.

We should point out that from the mathematical point of view nonnegative solutions of (1.1) are rare to find. This is what makes our model of study useful. In Theorem 4.2, we presented the simple case of a sustainable debt provided that all future surpluses are exactly equal to debt interest. Next, we present the case, in which future surpluses are greater than debt interest. Intuitively, debt must be sustainable in this case. However, in this case, the initial study model, (1.1), is not satisfied by the debt sequence $\left\{B_{n}\right\}$, for all future values of $n$. In this case, the debt behavior can only be described by our model of study.

Theorem 4.4. Assume that $\left\{\left(B_{n}, F_{n}\right)\right\}$ is a solution of System (2.8) and that:

(i) The initial debt value, $B_{0}$, is an arbitrary positive real number and that the initial value of savings, $F_{0}$ is equal to zero. That is,

$$
B_{0} \in(0, \infty) \text { and } F_{0}=0 \text {. }
$$

(ii) $\left\{r_{n}\right\}$ is an arbitrary future sequence representing interest rate, such that, for all nonnegative integer values of $n$,

$$
r_{n} \in(0,1) \text { and } \liminf _{n \rightarrow \infty} r_{n}>0
$$


(iii) For all n, there is a positive surplus which is greater than national debt interest. That is,

$$
S_{n+1}>r_{n} B_{n}, \text { for all } n \geq 0 \text {. }
$$

Then all debt values, $B_{n}$, current and future, are sustainable. In particular,

$$
0<B_{n}<P V_{n} \leq P V_{n}+F_{n}, \text { for all } n \geq 0
$$

and there exists a positive integer, $N=N\left(B_{0}, r_{0}, \ldots, r_{N}, S_{1}, \ldots, S_{N+1}\right)$, such that

$$
\begin{gathered}
0<B_{N} \leq B_{N-1} \leq \ldots \leq B_{0}, \quad F_{N}=F_{N-1}=\ldots=F_{0}=0 \\
B_{n}=B_{N}, \quad F_{n}=\left(1+r_{n-1}\right) F_{n-1}+S_{n}-r_{n-1} B_{N}, \text { for all } n>N, \text { and } F_{n} \uparrow \infty .
\end{gathered}
$$

Proof. The proof is straightforward and the details are omitted.

A quite simple case scenario, is the one in which, interest rates and surpluses are constant for all future times, that is,

$$
r_{n}=r, \quad S_{n}=S, \text { for all } n \geq 0, \quad r \in(0,1) \text { and } S \in(0, \infty) .
$$

In this case, the present value of all future surpluses, for all values of $n$ is: $P V_{n}=\frac{S}{r}$. The next theorem presents debt sustainability condition and future debt behavior.

Theorem 4.5. Assume that (4.3) holds. Also,

$$
B_{0} \in(0, \infty) \text { and } F_{0}=0 \text {. }
$$

Then, $P V_{n}=\frac{S}{r}$, for all values of $n$ and all debt values, $B_{n}$, current and future, are sustainable, if and only if,

$$
B_{0} \leq \frac{S}{r}
$$

In addition,

(i) if $B_{0}<\frac{S}{r}$, then $B_{n}<P V_{n}=\frac{S}{r}$, for all values of $n$ and there exists a positive integer $N$, such that

$$
\begin{gathered}
0<B_{N} \leq B_{N-1} \leq \ldots \leq B_{0}, \quad F_{N}=F_{N-1}=\ldots=F_{0}=0 \\
B_{n}=B_{N}, \quad F_{n}=\left(1+r_{n-1}\right) F_{n-1}+S_{n}-r_{n-1} B_{N}, \text { for all } n>N, \text { and } F_{n} \uparrow \infty .
\end{gathered}
$$

(ii) If $B_{0}>P V_{0}=\frac{S}{r}$, then

$$
B_{n}>P V_{n}=\frac{S}{r}, \quad B_{n+1}>B_{n} \text { and } F_{n}=0 \text { for all } n \geq 0, \text { and } \lim _{n \rightarrow \infty} B_{n}=\infty \text {. }
$$


(iii) If $B_{0}=\frac{S}{r}$, then

$$
B_{n}=P V_{n}=\frac{S}{r} \text { and } F_{n}=0 \text { for all } n \geq 0 .
$$

So far, in this section, we have assumed that there is always a positive surplus which services debt. However, there exist many cases, in which this is not true. For example for some future stages $n$, debt might be serviced by surplus, while for some other future $n$ it might not. Such a mixed type behavior might appear in the case where the behavior of the interest rates and surplus is periodic. Next, we present the debt sustainability condition and the future behavior of debt and savings under the assumption that future interest rates and surplus are periodic of period $q$. This case contains the scenario, where for some time periods, surplus is too low to sufficiently service debt or the case where deficit appears instead of surplus. Set

$$
k=\prod_{t=0}^{q-1}\left(1+r_{t}\right) \text { and } \Phi=\sum_{j=0}^{q-1} \frac{S_{j+1}}{\prod_{t=0}^{j}\left(1+r_{t}\right)} .
$$

Theorem 4.6. Assume that:

(i) The initial debt value, $B_{0}$, is an arbitrary positive real number and that the initial value of savings, $F_{0}$ is equal to zero. That is,

$$
B_{0} \in(0, \infty) \text { and } F_{0}=0 .
$$

(ii) $\left\{r_{n}\right\}$ is an arbitrary periodic sequence representing future interest rates, such that, for all nonnegative integer values of $n$,

$$
r_{n} \in(0,1) \text { and } r_{n q+m-1}=r_{m-1}, \quad m \in\{0,1 \ldots, q-1\} .
$$

(iii) For all $n \geq 0$,

$$
S_{n q+m}=S_{m}, \quad m \in\{0,1 \ldots, q-1\} .
$$

Then all debt values, $B_{n}$, current and future, are sustainable, if and only if,

$$
0<B_{0} \leq P V_{0}=\frac{\Phi k}{k-1} .
$$

Furthermore, the following are true:

(i) If

$$
B_{0}=P V_{0},
$$

then both debt and savings are periodic with period $q$. 
(ii) If

$$
B_{0}<P V_{0}
$$

then

$$
\lim _{n \rightarrow \infty} F_{n}=\infty \text { and } \lim _{n \rightarrow \infty} B_{n}=B_{N}, \text { for some } N \geq 0 .
$$

(iii) If

$$
B_{0}>P V_{0}
$$

then

$$
\lim _{n \rightarrow \infty} F_{n}=0 \text { and } \lim _{n \rightarrow \infty} B_{n}=\infty .
$$

Proof. The proof is straightforward and the details are omitted.

\section{Samuelson-Hicks Model}

In this section we present some examples related to the well known Samuelson-Hicks model, see [12]. We assume that, taxation rate, $\tau_{m}$, and government spending are both constants, that is, $\tau_{n}=\tau \in(0,1)$ and $G_{n}=G>0$, for all $n$. In addition, the values of the national income sequence $\left\{Y_{n}\right\}$, satisfy the Samuelson-Hicks model,

$$
Y_{n+2}=(b+\phi) Y_{n+1}-\phi Y_{n}+G, \quad n=0,1, \ldots,
$$

where, $b \in(0,1)$, is the marginal propensity of consumption, $\phi=b k \in(0, \infty)$, where, $k$, is the investment acceleration coefficient, $G \in(0, \infty)$, and the initial values of national income, $Y_{0}, Y_{1}$ are arbitrary positive numbers. For the simplicity of our example, we also assume that the interest rate is constant, that is, $r_{n}=r \in(0,1)$, for all $n$. In view of, $S_{n+1}=\tau Y_{n}-G$, we have

$$
S_{n+3}=(b+\phi) S_{n+2}-\phi S_{n+1}+(\tau+b-1) G, \quad n=0,1, \ldots .
$$

The equilibrium point of (5.2) is $\bar{S}=\frac{(b+\tau-1) G}{1-b}$. The corresponding characteristic equation, $\lambda^{2}-(b+\phi) \lambda+\phi=0$, has two real distinct solutions,

$$
\lambda_{1}=\frac{b+\phi-\sqrt{(b+\phi)^{2}-4 \phi}}{2}, \quad \lambda_{2}=\frac{b+\phi+\sqrt{(b+\phi)^{2}-4 \phi}}{2},
$$

if and only if, $|\sqrt{\phi}-1|>\sqrt{1-b}$. If $|\sqrt{\phi}-1|<\sqrt{1-b}$, there exist two complex eigenvalues, $\lambda_{1}=\sqrt{\phi} e^{-i \theta}$ and $\lambda_{2}=\sqrt{\phi} e^{i \theta}$, where $\theta=\arctan \frac{\sqrt{4 \phi-(\phi+b)^{2}}}{b+\phi} \in$ $\left(0, \frac{\pi}{2}\right)$. Finally, if $|\sqrt{\phi}-1|=\sqrt{1-b}$, then $\lambda_{1}=\lambda_{2}=\sqrt{\phi} \in(0,1) \cup(1,2)$. We should point out that the two characteristic roots, $\lambda_{1}, \lambda_{2}$, lie inside the unit circle, if and only if, $\phi<1$. If $\phi=1$, both characteristic roots are located on the boundary of the unit circle. As it turns out, this is the most interesting case. 


\subsection{Debt Sustainability Conditions when GDP is Globally Asymp- totically Stable}

Assume that

$$
\phi<1 \text {. }
$$

The equilibrium point of (5.2), is globally asymptotically stable. We consider the following cases.

\subsubsection{Case 1: Real Distinct Eigenvalues}

First, we consider the case in which both eigenvalues $\lambda_{1}, \lambda_{2}$ are real and distinct. In particular, in this case both eigenvalues lie within the interval $(0,1)$.

Theorem 5.1. Assume that

$$
0<\sqrt{\phi}<1-\sqrt{1-b}
$$

Then debt is sustainable, if and only if,

$$
B_{0} \leq P V_{0}=\frac{c_{1}}{1+r-\lambda_{1}}+\frac{c_{2}}{1+r-\lambda_{2}}+\frac{\bar{S}}{r},
$$

where

$$
c_{1}=\frac{\lambda_{2}\left(S_{2}-\bar{S}\right)-\left(S_{1}-\bar{S}\right)}{\lambda_{2}-\lambda_{1}} \text { and } c_{2}=\frac{\left(S_{1}-\bar{S}\right)-\lambda_{1}\left(S_{2}-\bar{S}\right)}{\lambda_{2}-\lambda_{1}} .
$$

Furthermore, the long term debt behavior is as follows:

(i) If $B_{0}<\frac{c_{1}}{1+r-\lambda_{1}}+\frac{c_{2}}{1+r-\lambda_{2}}+\frac{\bar{S}}{r}$, then

$$
B_{n}=B_{N}, \text { for some } N \geq 0 \text { and } \lim _{n \rightarrow \infty} F_{n}=\infty \text {. }
$$

(ii) If $B_{0}=\frac{c_{1}}{1+r-\lambda_{1}}+\frac{c_{2}}{1+r-\lambda_{2}}+\frac{\bar{S}}{r}$, then

$$
B_{n}=P V_{n}=\frac{c_{1} \lambda_{1}^{n}}{1+r-\lambda_{1}}+\frac{c_{2} \lambda_{2}^{n}}{1+r-\lambda_{2}}+\frac{\bar{S}}{r} \rightarrow \frac{\bar{S}}{r}, \text { and } F_{n}=0, \text { for all } n \geq 0 \text {. }
$$

(iii) If $B_{0}>\frac{c_{1}}{1+r-\lambda_{1}}+\frac{c_{2}}{1+r-\lambda_{2}}+\frac{\bar{S}}{r}$, then

$$
B_{n} \rightarrow \infty \text { and } F_{n}=0, \text { for all } n \geq 0 \text {. }
$$

In Figure 5.1, we present graphically a numerical example of a stabilized behavior of debt to GDP ratio when the values $r_{n}$ of the interest rate sequence, are constantly equal with a positive value $r$ and Condition (5.3) holds. Both ratios converge eventually monotonically. 


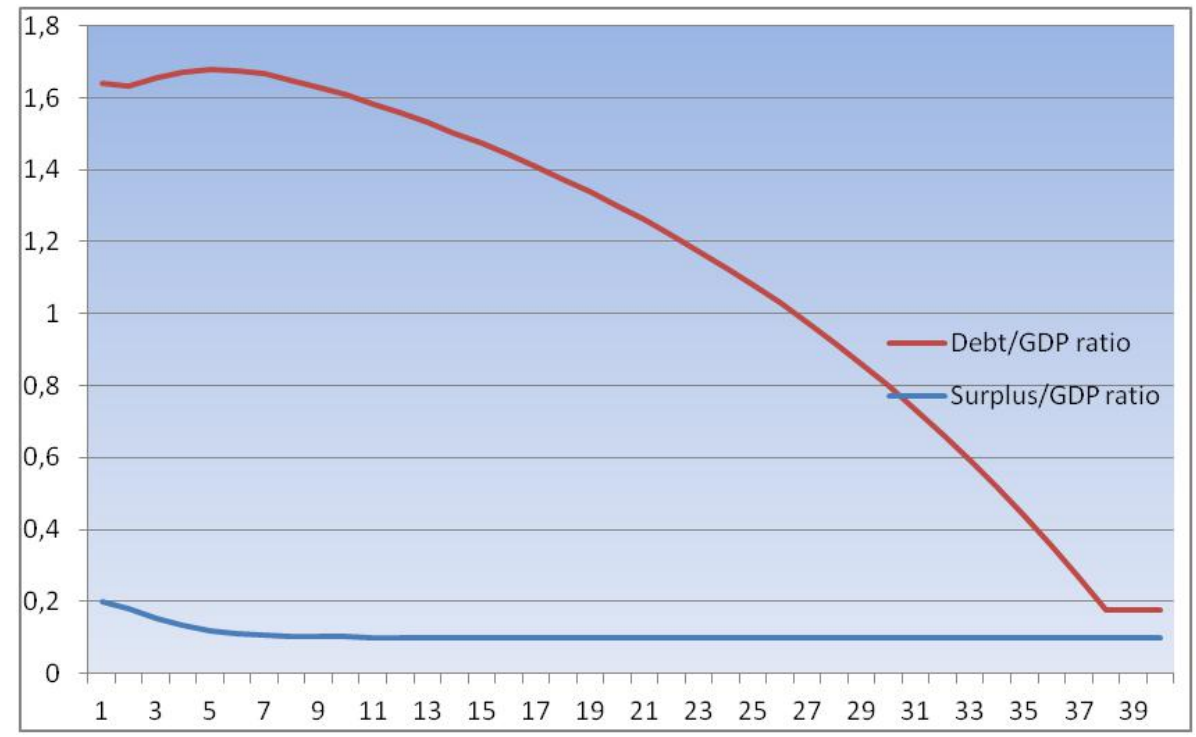

Figure 5.1: Stabilization of Surplus/GDP and Debt/GDP ratios, when the two eigenvalues of the characteristic equation of surplus and GDP equations are positive, and debt sustainability condition holds.

\subsubsection{Case 2: Double Eigenvalue}

In this case $\lambda_{1}=\lambda_{2}=\sqrt{\phi} \in(0,1)$.

Theorem 5.2. Assume that

$$
\sqrt{\phi}=1-\sqrt{1-b}
$$

Then debt is sustainable, if and only if,

$$
B_{0} \leq P V_{0}=\frac{c_{1}}{1+r-\sqrt{\phi}}+\frac{c_{2} \sqrt{\phi}}{(1+r-\sqrt{\phi})^{2}}+\frac{\bar{S}}{r}
$$

where

$$
c_{1}=S_{1}-\bar{S} \text { and } c_{2}=\frac{\left(S_{2}-\bar{S}\right)-\sqrt{\phi}\left(S_{1}-\bar{S}\right)}{\sqrt{\phi}} .
$$

Furthermore, the long term debt behavior is as follows:

(i) If $B_{0}<\frac{c_{1}}{1+r-\sqrt{\phi}}+\frac{c_{2} \sqrt{\phi}}{(1+r-\sqrt{\phi})^{2}}+\frac{\bar{S}}{r}$, then

$$
B_{n}=B_{N}, \text { for some } N \geq 0 \text { and } \lim _{n \rightarrow \infty} F_{n}=\infty \text {. }
$$

(ii) If $B_{0}=\frac{c_{1}}{1+r-\sqrt{\phi}}+\frac{c_{2} \sqrt{\phi}}{(1+r-\sqrt{\phi})^{2}}+\frac{\bar{S}}{r}$, then

$$
B_{n}=P V_{n}=\frac{c_{1} \sqrt{\phi}^{n}}{1+r-\sqrt{\phi}}+\frac{c_{2} \sqrt{\phi}^{n+1}}{(1+r-\sqrt{\phi})^{2}}+\frac{\bar{S}}{r} \rightarrow \frac{\bar{S}}{r}, \text { and } F_{n}=0 \text {, for all } n \geq 0 \text {. }
$$


(iii) If $B_{0}>\frac{c_{1}}{1+r-\sqrt{\phi}}+\frac{c_{2} \sqrt{\phi}}{(1+r-\sqrt{\phi})^{2}}+\frac{\bar{S}}{r}$, then

$$
B_{n} \rightarrow \infty \text { and } F_{n}=0 \text {, for all } n \geq 0 \text {. }
$$

\subsubsection{Case 3: Complex Eigenvalues}

In the case, where

$$
1-\sqrt{1-b}<\sqrt{\phi}<1
$$

both eigenvalues of the characteristic equation of (5.2) are complex. More precisely,

$$
\lambda_{1}=\sqrt{\phi} e^{-i \theta}, \quad \lambda_{2}=\sqrt{\phi} e^{i \theta},
$$

where $\theta=\arctan \frac{\sqrt{4 \phi-(\phi+b)^{2}}}{b+\phi} \in\left(0, \frac{\pi}{2}\right)$. Moreover, $\lambda_{1}=\lambda_{2}=\sqrt{\phi}<1$. The equilibrium $\bar{S}$ of (5.2) is globally asymptotically stable and solutions converge to the equilibrium in an oscillatory fashion.

Theorem 5.3. Assume that

$$
1-\sqrt{1-b}<\sqrt{\phi}<1 .
$$

Then debt is sustainable, if and only if,

$$
B_{0} \leq P V_{0}=\frac{c_{1}((1+r)-\sqrt{\phi} \cos \theta)+c_{2} \sqrt{\phi} \sin \theta}{(1+r)^{2}+\phi-2(1+r) \sqrt{\phi} \cos \theta}+\frac{\bar{S}}{r}
$$

where

$$
c_{1}=S_{1}-\bar{S} \text { and } c_{2}=\frac{\left(S_{2}-\bar{S}\right)-\cos \theta \sqrt{\phi}\left(S_{1}-\bar{S}\right)}{\sin \theta \sqrt{\phi}} .
$$

Furthermore, the long term debt behavior is as follows:

(i) If $B_{0}<P V_{0}$, then

$$
B_{n}=B_{N}, \text { for some } N \geq 0 \text { and } \lim _{n \rightarrow \infty} F_{n}=\infty .
$$

(ii) If $B_{0}=P V_{0}$, then

$$
\begin{gathered}
B_{n}=P V_{n} \\
=\frac{c_{1}[(1+r) \cos n \theta-\sqrt{\phi} \cos (n-1) \theta]+c_{2}[(1+r) \sin n \theta+\sqrt{\phi} \sin (n-1) \theta]}{(1+r)^{2}+\phi-2(1+r) \sqrt{\phi} \cos \theta} \cdot(\sqrt{\phi})^{n} \\
+\frac{\bar{S}}{r} \rightarrow \frac{\bar{S}}{r}, \text { and } F_{n}=0, \text { for all } n \geq 0 .
\end{gathered}
$$

(iii) If $B_{0}>P V_{0}$, then

$$
B_{n} \rightarrow \infty \text { and } F_{n}=0, \text { for all } n \geq 0 .
$$

In Figure 5.2, we present an example of exploding debt/GDP ratio and converging to zero surplus/GDP ratio, when the values of the interest rate sequence $r_{n}$ are constant, Condition (5.5) holds, and $B_{0}>S$. 


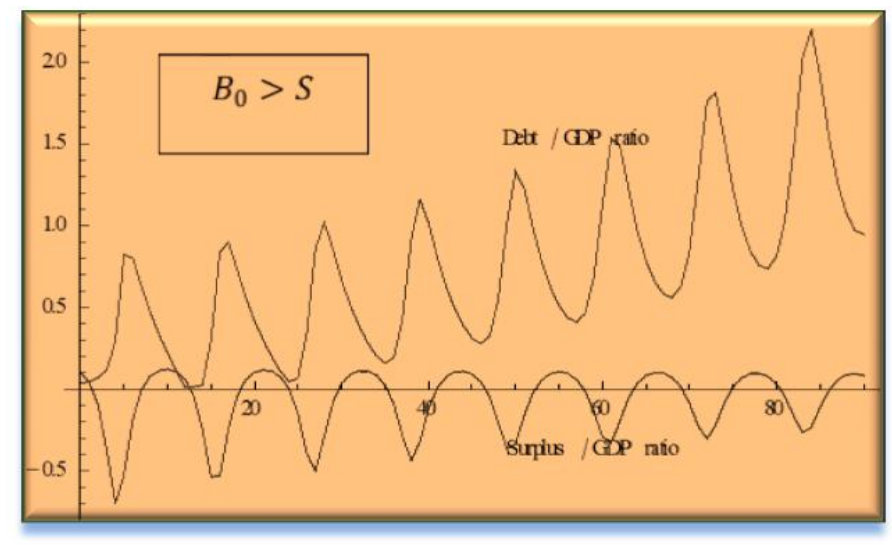

Figure 5.2: Exploding debt/GDP ratio and converging to zero surplus/GDP ratio, when the two eigenvalues of the characteristic equation of the surplus and GDP equation are complex, and debt sustainability condition is violated.

\subsubsection{Case 4: Debt Sustainability when GDP is Neutrally Stable}

If

$$
\phi=1
$$

(5.2) becomes

$$
S_{n}=(b+1) S_{n-1}-S_{n-2}+(\tau+b-1) G, \quad n=0,1, \ldots .
$$

In this case, both eigenvalues of the characteristic equation of (5.2) are on the unit circle with magnitude equal to one. They are:

$$
\lambda_{1}=\frac{b+1-i \sqrt{4-(b+1)^{2}}}{2} \text { and } \lambda_{2}=\frac{b+1+i \sqrt{4-(b+1)^{2}}}{2} .
$$

In polar form, $\lambda_{1}=e^{-i \theta}, \quad \lambda_{2}=e^{i \theta}$,

$$
\theta=\arctan \frac{\sqrt{4-(1+b)^{2}}}{b+1} \in\left(0, \frac{\pi}{2}\right) \text {. }
$$

The solution $\left\{S_{n}\right\}_{n=-2}^{\infty}$, of (5.6) is given by

$$
\left.\begin{array}{l}
S_{n}=\bar{S}+c_{1} \cos (n+2) \theta+c_{2} \sin (n+2) \theta \\
c_{1}=S_{-2}-\bar{S} \text { and } c_{2}=\frac{2\left(S_{-1}-\bar{S}\right)-(b+1)\left(S_{-2}-\bar{S}\right)}{\sqrt{4-(b+1)^{2}}}
\end{array}\right\} .
$$

The following lemma will be useful in the sequel.

Lemma 5.4. Let $\theta$ be the angle given by (5.7). Then

$$
b=\frac{2-\sqrt{\tan ^{2} \theta+1}}{\sqrt{\tan ^{2} \theta+1}} \in(0,1) \Leftrightarrow \theta=\omega \pi, \omega \in\left(0, \frac{1}{3}\right) .
$$


Proof. The proof follows from straightforward calculations and the details are omitted.

The next theorem, known as Kronecker's approximation theorem will be useful in the sequel. For the proof of the theorem see [4].

Theorem 5.5 (See [4, Theorem 439 on page 364]). If $\rho$ is an irrational number, the infinite sequence $\{\rho \cdot t-\lfloor\rho \cdot t\rfloor\}_{t=1}^{\infty}$ is dense in the interval $(0,1)$.

Theorem 5.6. Let $\left\{S_{n}\right\}_{n=-2}^{\infty}$ be a solution of (5.6). Let $\theta$ be the angle given by (5.7). Then the following statements are true:

(i) If $\theta=\omega \pi$, where $0<\omega<\frac{1}{3}$, is irrational, then the solution $\left\{S_{n}\right\}_{n=-2}^{\infty}$ of 5.2, is dense within a subset of the interval $\left(\bar{S}-S^{* *}, \bar{S}+S^{* *}\right)$

(ii) If $\theta=\omega \pi, 0<\omega=\frac{l}{m}<\frac{1}{3}$, and l, $m$ are positive integers, then the solution $\left\{S_{n}\right\}_{n=-2}^{\infty}$ of (5.2), is periodic with period $2 \mathrm{~m}$.

Proof. The proof follows directly from the periodic character of sin and cos, and Theorem 5.5. The proof is complete.

In Figure 5.3, we present the diagram of fluctuating debt ratio behavior versus surplus, which is also fluctuating when, the values of the interest rate sequence $r_{n}$ are constant, $\phi=1, \theta=\omega \pi$, where $\omega \in\left(0, \frac{1}{3}\right)$ is an arbitrary irrational number. The fluctuating behavior of both ratios is "chaotic", in the sense that the set of values of both sequences is dense within a certain interval.

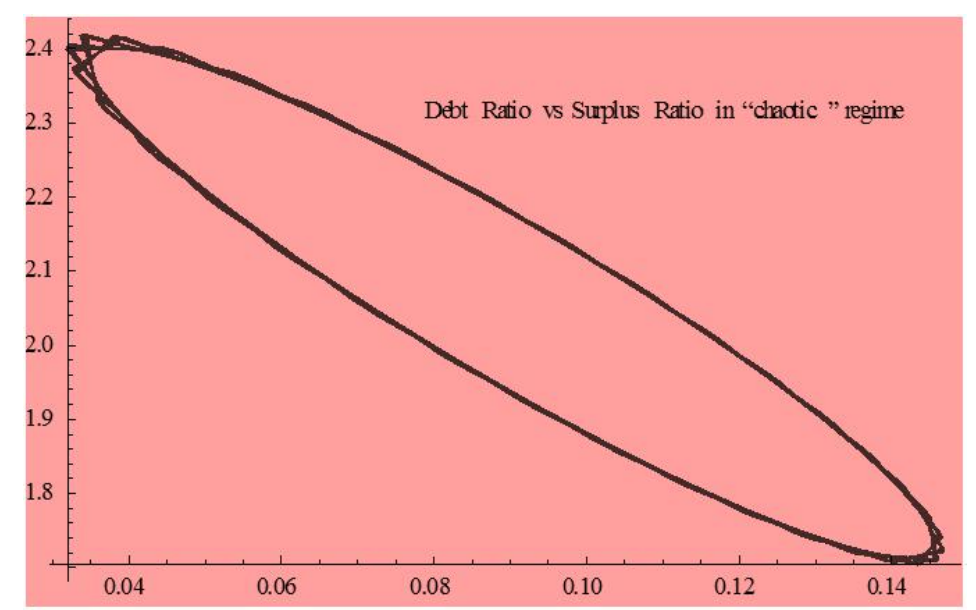

Figure 5.3: No periodic behavior. Debt and Surplus Ratios assume all values within a certain interval. Debt is sustainable.

In Figure 5.4, we present the time series version of the diagram presented in Figure 5.3 . 


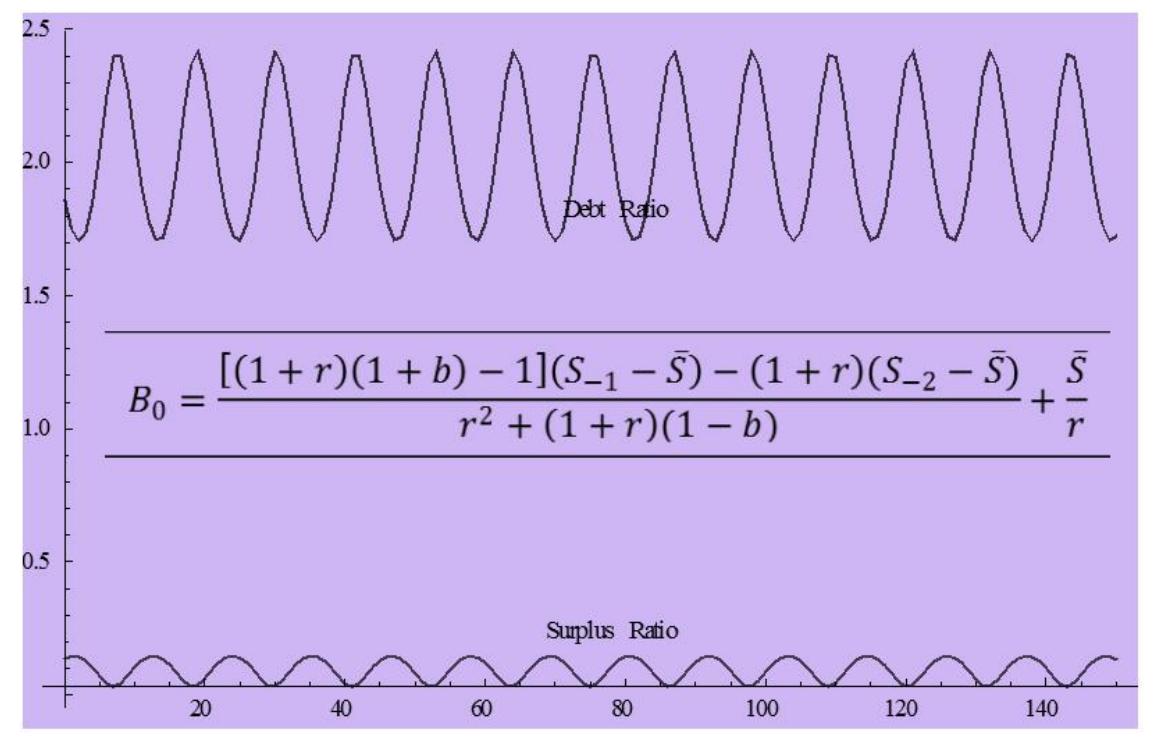

Figure 5.4: Time series representation of the graph presented in Figure 3. GDP and surplus ratio fluctuation in the chaotic regime.

\subsubsection{Case 4a: Debt Sustainability when GDP behavior is periodic}

In this case, together with the debt sustainability conditions we establish necessary and sufficient conditions such that the debt and savings sequences $\left\{B_{n}\right\}$ and $\left\{F_{n}\right\}$, respectively, are periodic.

Theorem 5.7. Assume that $\phi=1$. Assume that the angle $\theta$ presented in (5.7), is

$$
\theta=\frac{l}{m} \cdot \pi, \quad 0<\frac{l}{m}<\frac{1}{3}
$$

where $l, m$ are positive integers. Let $c_{1}$ and $c_{2}$ be the constants presented in (5.8). $\left\{S_{n}\right\}_{n=-2}^{\infty}$ is a solution of (5.6), with initial conditions $S_{-2}$ and $S_{-1}$, such that

$$
\Phi_{0}=\sum_{j=0}^{q-1} \frac{s_{j}}{(1+r)^{j}}>0, \quad q=2 m
$$

Set

$$
B_{0}>0, \quad F_{0}=0
$$

and let $\left\{\left(B_{n}, F_{n}\right)\right\}_{n=0}^{\infty}$ and $\left\{B_{n}-F_{n}\right\}_{n=0}^{\infty}$ be solutions of System (2.8) and (2.7), with

$$
r_{n}=r \in(0,1), \text { for all } n \geq 0 \text {. }
$$

Then the following statements are true: 
(i) If

$$
B_{0}=\frac{\Phi_{0}(1+r)^{q}}{(1+r)^{q}-1},
$$

then the debt sequence is periodic with period $q=2 \mathrm{~m}$.

(ii) If

$$
B_{0}<\frac{\Phi_{0}(1+r)^{q}}{(1+r)^{q}-1}
$$

then

$$
\lim _{n \rightarrow \infty} F_{n}=\infty \text { and } \lim _{n \rightarrow \infty} B_{n}=B_{N} \text {, for some } N \geq 0 .
$$

(iii) If

$$
B_{0}>\frac{\Phi_{0}(1+r)^{q}}{(1+r)^{q}-1}
$$

then

$$
\lim _{n \rightarrow \infty} F_{n}=0 \text { and } \lim _{n \rightarrow \infty} B_{n}=\infty .
$$

Proof. (i) It suffices to show that

$$
B_{q}=B_{0} \text { and } F_{q}=F_{0}=0 .
$$

The solution of (2.7), is given by

$$
B_{n}-F_{n}=(1+r)^{n}\left(B_{0}-\sum_{j=0}^{n-1} \frac{S_{j}}{(1+r)^{j+1}}\right), n=0,1, \ldots .
$$

By Theorem 5.6, the solution $\left\{S_{n}\right\}$ of (5.2), is periodic of period $q$. Substituting, $n=q$, into the last equation, we have

$$
B_{q}-F_{q}=(1+r)^{q}\left(B_{0}-\Phi_{0}\right)=B_{0}>0,
$$

and so, one can easily see that

$$
B_{q}=B_{0} \text { and } F_{q}=F_{0}=0 .
$$

(ii) In a similar fashion as in $(i)$, we have

$$
B_{q}-F_{q}=(1+r)^{q}\left(B_{0}-\Phi_{0}\right)<B_{0}-F_{0}=B_{0}<\frac{\Phi_{0}(1+r)^{q}}{(1+r)^{q}-1} .
$$

Also,

$$
B_{q+1}=F_{q+1}=(1+r)\left(B_{q}-F_{q}\right)-S_{q}<(1+r)\left(B_{0}-F_{0}\right)-S_{0}=B_{1}-F_{1} .
$$


By using induction, it can be easily seen that, for all $n \geq 0$,

$$
B_{(n+1) q+j}-F_{(n+1) q+j}<B_{n q+j}-F_{n q+j}, \quad j=0,1, \ldots, q-1 .
$$

Thus, for each $j=0,1, \ldots, q-1$, the corresponding sequence $\left\{B_{n q+j}\right\}_{n=0}^{\infty}$, is strictly decreasing and so,

$$
\lim _{n \rightarrow \infty}\left(B_{n q+j}-F_{n q+j}\right)=\Psi_{j} \in[-\infty, \infty) .
$$

In particular, let us consider the sequence $\left\{B_{n q}\right\}_{n=0}^{\infty}$. For this sequence, we see that

$$
\lim _{n \rightarrow \infty}\left(B_{n q}-F_{n q}\right)=\Psi_{0} \in\left[-\infty, \frac{\Phi_{0}(1+r)^{q}}{(1+r)^{q}-1}\right) .
$$

We claim that

$$
\Psi_{0}=-\infty .
$$

Assume for the sake of contradiction, that

$$
\Psi_{0} \in\left(-\infty, \frac{\Phi_{0}(1+r)^{q}}{(1+r)^{q}-1}\right)
$$

and observe, that the sequence

$$
\Psi_{0}, \Psi_{1}, \ldots, \Psi_{q-1}, \Psi_{0}, \Psi_{1}, \ldots, \Psi_{q-1}, \ldots,
$$

is a periodic solution of the equation

$$
x_{n+1}=(1+r) x_{n}-S_{n}, \quad n=0,1, \ldots, .
$$

Thus,

$$
\Psi_{0}=(1+r)^{q}\left(\Psi_{0}-\Phi_{0}\right),
$$

from which it follows that

$$
\Psi_{0}=\frac{\Phi_{0}(1+r)^{q}}{(1+r)^{q}-1}
$$

a contradiction. Similarly, it can be shown that

$$
\lim _{n \rightarrow \infty}\left(B_{n k+j}-F_{n k+j}\right)=-\infty, \quad j=1, \ldots, q-1,
$$

and so,

$$
\lim _{n \rightarrow \infty}\left(B_{n}-F_{n}\right)=-\infty .
$$

Thus, there exists $N>0$, such that

$$
B_{n}-F_{n}<0, \text { for all } n \geq N \text {. }
$$

(iii) The proof in this case, is along the same lines, with the proof presented in $(i i)$ and the details are omitted. 
The results of Theorem 5.7 can be easily extended to the following theorem, in which we assume that the interest rate sequence $\left\{r_{n}\right\}$ is periodic with the same period $q$, as the sequence $\left\{S_{n}\right\}$.

Theorem 5.8. Assume that $\phi=1$. Assume that the angle $\theta$ presented in (5.7), is

$$
\theta=\frac{l}{m} \cdot \pi, \quad 0<\frac{l}{m}<\frac{1}{3}
$$

where $l, m$ are positive integers. Let $c_{1}$ and $c_{2}$ be the constants presented in (5.8). $\left\{S_{n}\right\}_{n=-2}^{\infty}$ is a solution of (5.6), with initial conditions $S_{-2}$ and $S_{-1}$, such that

$$
\Phi_{0}=\sum_{j=0}^{q-1} \frac{s_{j}}{\prod_{t=0}^{j}\left(1+r_{t}\right)}>0, \quad q=2 m .
$$

Set

$$
B_{0}>0, \quad F_{0}=0
$$

and let $\left\{\left(B_{n}, F_{n}\right)\right\}_{n=0}^{\infty}$ and $\left\{B_{n}-F_{n}\right\}_{n=0}^{\infty}$ be solutions of System (2.8) and (2.7), with

$$
r_{n+q}=r_{n} \in(0,1), \text { for all } n \geq 0
$$

Then the following statements are true:

(i) If

$$
B_{0}=\frac{\Phi_{0} \prod_{j=0}^{q-1}\left(1+r_{j}\right)}{\prod_{j=0}^{q-1}\left(1+r_{j}\right)-1},
$$

then the debt sequence is periodic with period $q=2 \mathrm{~m}$.

(ii) If

$$
B_{0}<\frac{\Phi_{0} \prod_{j=0}^{q-1}\left(1+r_{j}\right)}{\prod_{j=0}^{q-1}\left(1+r_{j}\right)-1}
$$

then

$$
\lim _{n \rightarrow \infty} F_{n}=\infty \text { and } \lim _{n \rightarrow \infty} B_{n}=B_{N}, \text { for some } N \geq 0
$$

(iii) If

$$
B_{0}>\frac{\Phi_{0} \prod_{j=0}^{q-1}\left(1+r_{j}\right)}{\prod_{j=0}^{q-1}\left(1+r_{j}\right)-1}
$$

then

$$
\lim _{n \rightarrow \infty} F_{n}=0 \text { and } \lim _{n \rightarrow \infty} B_{n}=\infty .
$$


Remark 5.9. Although it is stated, in both theorems of this section, that the length of an arbitrary periodic cycle is equal with $2 m$, we should mention that there exist periodic cycles of length $m$. In fact, if $\theta=\frac{2 k}{m} \cdot \pi$, then the corresponding solutions are periodic of prime period $m$. In addition, in view of the fact that $\theta \in\left(0, \frac{\pi}{3}\right)$, the minimum length of a periodic cycle is seven. Also, it must be mentioned that for each positive integer $j$, greater than or equal to seven, there exists a periodic cycle of prime period $j$.

In Figure 5.5, we present an example of perfectly periodic behavior of debt ratio vs perfect periodic behavior of surplus ratio when $\phi=1$ and Condition (5.11) holds. The length of the period is the minimum possible equal with seven. To achieve such periodic solution we have chosen $\theta=\frac{2 \pi}{7}$.

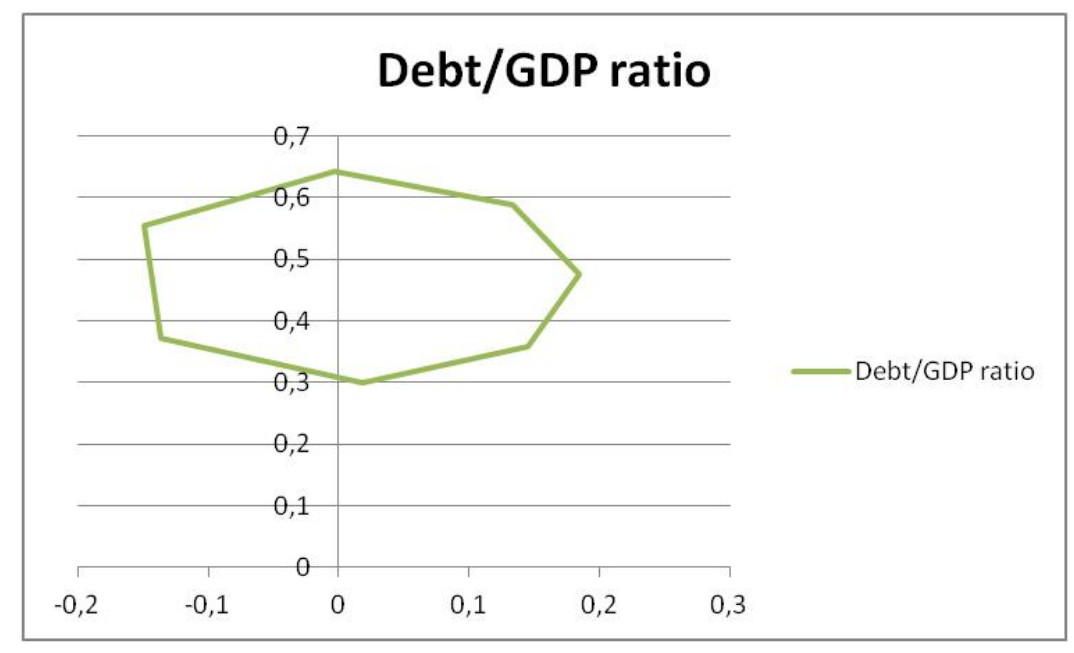

Figure 5.5: Example

In Figure 5.6, we present the time series version of the diagram presented in Figure 5.5 .

In Figure 5.7, we present the behavior of debt and surplus ratios when, $\phi=1$, Condition (5.12) holds, and $\theta=\frac{2 \pi}{7}$. The behavior of surplus ratio in this case is perfectly periodic. However, the behavior of debt ratio is eventually periodic. 


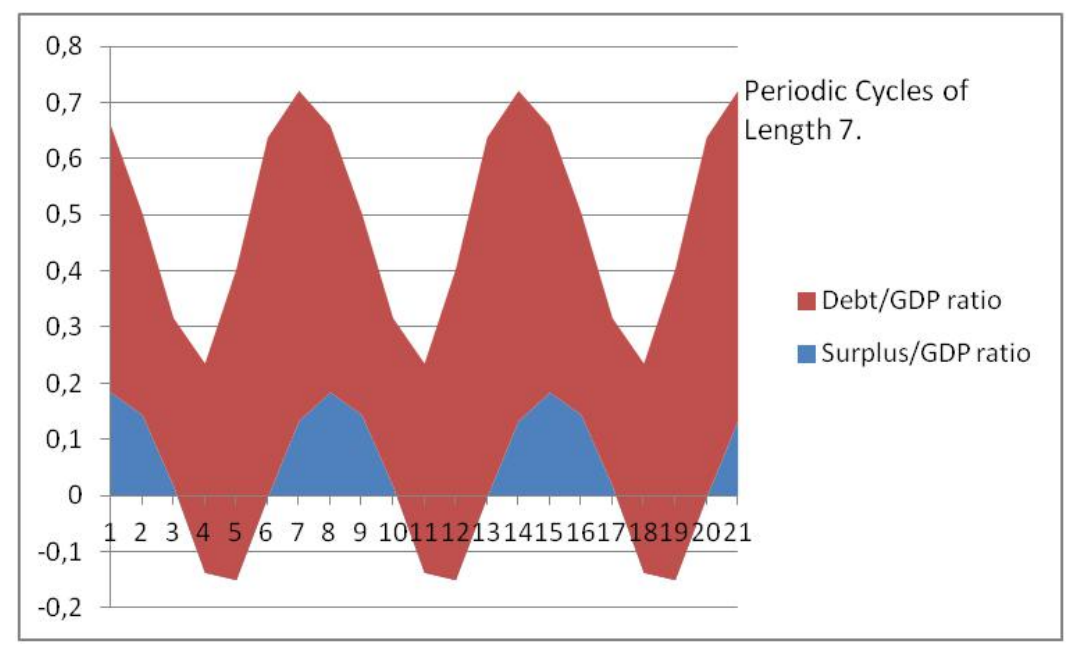

Figure 5.6: Time series version

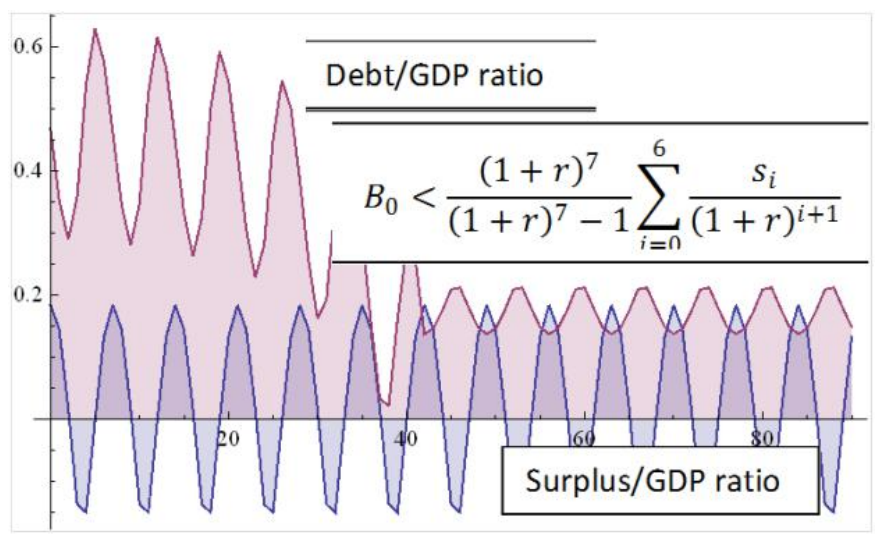

Figure 5.7: Behavior of debt and surplus ratios

\section{References}

[1] R. J. Barro, Are Government Bonds Net Wealth? Journal of Political Economy, (82):1095-1117, 1974.

[2] R. J. Barro, On the determination of the public debt. Journal of Political Economy, (87):940-971, 1979.

[3] E. Camouzis and G. Ladas, Dynamics of Third-Order Rational Difference Equations; With Open Problems and Conjectures, Chapman \& Hall/CRC Press, November 2007.

[4] Hardy and Wright, The Theory of Numbers, Oxford, 1938. 
[5] M. A. Friedman, A Theory of the Consumption Function, Princeton University Press, 1957.

[6] Gilchrist, John and Charles Himmelberg, Evidence on the role of cash flow for investment, Journal of Monetary Economics 36, 541-572, 1995.

[7] Holmstrom, Bengt, and Jean Tirole, Private and Public Supply of Liquidity, Journal of Political Economy 106, 1-40, 1997.

[8] J. M. Keynes, The General Theory of Employment, Interest, and Money, Harcourt, Brace, 1936.

[9] Ilias Kostarakos-Stelios Kotsios, Feedback policy rules for government spending: an algorithmic approach, Economic Structures (2017) 6: 5.

[10] Stelios Kotsios-Kostarakos Ilias. Controlling National Income and Debt via Extra Taxation. A Model Matching Algorithmic Approach, Vestnik of Saint Petersburg University. Series 10. Applied mathematics. Computer science. Control processes, 2016, issue 4, pp. 86-91.

[11] David Ricardo, "Essay on the Funding System" in The Works of David Ricardo. With a Notice on the Life and Writings of the Author, by J.T. McCulloch, London, John Murray, 1888.

[12] P. Samuelson, Interactions between the Multiplier Analysis and the Principle of Acceleration, Review of Economics and Statistics, 21 (2), 75-78, 1939.

[13] J.T. Sargent and N. Wallace, Some unpleasant monetaristic arithmetic, Federal reserve Bank of Minneapolis Quarterly Review, 5 3:1-17, 1981.

[14] J. D. Ostry, A. R. Ghosh, and R. Espinoza, When Should Public Debt be Reduced?, IMF Staff Discussion Note, June 2015. 
